6 Tomassetti S, Ruy JH, Gurioli C, et al. The effect of anticoagulant therapy for idiopathic pulmonary fibrosis in real life practice. Sarcoidosis Vasc Diffuse Lung Dis 2013; 30: 121-127.

7 Margaritopoulos GA, Antoniou KM. Can warfarin be used in the treatment of pulmonary embolism in idiopathic pulmonary fibrosis? Am J Respir Crit Care Med 2016; 193: 810-811.

8 Kreuter M, Ehlers-Tenenbaum S, Palmowski K, et al. Impact of comorbidities on mortality in patients with idiopathic pulmonary fibrosis. PLoS One 2016; 11: e0151425.

9 King TE Jr, Bradford WZ, Castro-Bernardini S, et al. A phase 3 trial of pirfenidone in patients with idiopathic pulmonary fibrosis. N Engl J Med 2014; 370: 2083-2092.

10 Noble PW, Albera C, Bradford WZ, et al. Pirfenidone in patients with idiopathic pulmonary fibrosis (CAPACITY): two randomised trials. Lancet 2011; 377: 1760-1769.

\title{
Cardiac safety of extensively drug-resistant tuberculosis regimens including bedaquiline, delamanid and clofazimine
}

\author{
To the Editor:
}

I read with interest the recent article by TADOLINI et al. [1] describing QT (ECG Q-T wave interval) prolongation in a patient with extensively drug-resistant tuberculosis (XDR-TB) being treated with both bedaquiline and delamanid. QT prolongation is a recognised safety concern for both these drugs. In a placebo-controlled trial of delamanid in multidrug resistant-TB, QTcF intervals (using the correction for heart rate of Fridericia [2]) increased $12.1 \mathrm{~ms}$ more from baseline in delamanid recipients over 6-10 weeks [3]. An increase of similar magnitude was observed in an open-label 24-week trial of bedaquiline, with most of the effect becoming apparent within the first 1-2 weeks [4]. The authors are correct that there is currently no clinical information regarding QT prolongation when these two drugs are co-administered.

However, readers may be less aware of QT prolongation due to clofazimine, which this patient also received as part of XDR-TB treatment. Clofazimine is a potent inhibitor of hERG potassium channel signalling, with essentially complete inhibition occurring in vitro at sub-therapeutic concentrations $[4,5]$. hERG mutations can result in QT prolongation and potentially fatal arrhythmias, including ventricular tachycardia and torsade de pointe [6,7]. In vitro measurement of hERG signalling effects is an important preclinical drug development tool to identify potential arrhythmia-inducing compounds [8], although recent studies indicate the modulating effects of other channels [9]. Testing for hERG signalling effects was not available at the time clofazimine was developed, some 50 years ago.

Two studies and one case report have confirmed the QT prolonging effects of clofazimine. In one study, 105 patients with newly diagnosed drug-sensitive TB were treated for 2 weeks with one of seven drugs or regimens ( $\mathrm{n}=15$ per arm), of which four included clofazimine $100 \mathrm{mg}$ daily [10]. By day 14, QT intervals in patients receiving clofazimine alone increased from baseline by a mean of $17 \mathrm{~ms}$. Patients receiving regimens containing both clofazimine and bedaquiline had increases of 20-21 ms. Patients receiving neither drug had no change in QT ( -3 to $2 \mathrm{~ms}$ ). Given the unusual pharmacokinetics of clofazimine, it is unlikely that this short treatment interval (14 days) was sufficient to reach steady state effects.

In a second study, 233 patients with drug-resistant TB were treated for 24 weeks with bedaquiline in an open label, single arm trial, in combination with an individualised background regimen [4]. 10 patients not receiving clofazimine at the time of enrolment were started on bedaquiline plus a background regimen including clofazimine. By week 24, their mean \pm SE QTcF interval had increased 41.5 $\pm 8.4 \mathrm{~ms}$ from baseline. In contrast, in patients whose background regimen did not include clofazimine, the QTcF interval increased by only $12.9 \pm 4.1 \mathrm{~ms}$ ( $\mathrm{p}=0.009)$.

Finally, one case report describes ventricular tachycardia and torsade de pointe occurring in a 66 year-old man who had been treated for recurrent erythema nodosum leprosum with $300 \mathrm{mg}$ of clofazimine per day for 11 months [11].

These findings indicate that clofazimine may indeed have been an important contributor to the QT prolongation in the patient reported by TADOLINI et al. [1]. Studies are needed to determine if clofazimine potentiates the combined QT prolonging effects of bedaquiline plus delamanid as it does with bedaquiline 
alone. The safety of regimens containing bedaquiline plus delamanid may be substantially improved if clofazimine can be omitted.

-

@ERSpublications

Clofazimine prolongs the QT interval and can potentiate the QT effects of other MDR-TB drugs http://ow.ly/70My302XuKm

Robert S. Wallis

Aurum Institute, Johannesburg, South Africa.

Correspondence: Robert S. Wallis, Aurum Institute, 29 Queens Rd, Parktown 2193, Johannesburg, South Africa.

E-mail: rwallis@auruminstitute.org

Received: June 192016 | Accepted after revision: July 262016

Conflict of interest: None declared.

\section{References}

1 Tadolini M, Lingtsang RD, Tiberi S, et al. First case of extensively drug-resistant tuberculosis treated with both delamanid and bedaquiline. Eur Respir J 2016; 48: 935-938.

2 Fridericia LS. The duration of systole in an electrocardiogram in normal humans and in patients with heart disease. 1920. Ann Noninvasive Electrocardiol 2003; 8: 343-351.

3 Deltyba summary of product characteristics. Report number WC500166232. European Medicines Agency 2015. www.ema.europa.eu/docs/en_GB/document_library/EPAR_-_Product_Information/human/002552/WC500166232. pdf Date last accessed: July 12, 2016.

4 Dannemann B, Bakare N, De Marez T, et al. QTcF prolongation in a phase II trial of TMC207 plus background regimen as treatment for MDR-TB: effect of co-administration of clofazimine. ICAAC 2012; 52: A1259.

5 Everitt D. Clofazimine in clinical trials for tuberculosis. Resurrecting clofazimine. www.resisttb.org/wp-content/ uploads/2013/08/Global-Alliance-Clinical-Work-with-Clofazimine.pdf Date last accessed: July 12, 2016.

6 Sanguinetti MC, Tristani-Firouzi M. hERG potassium channels and cardiac arrhythmia. Nature 2006; 440: 463-469.

7 Hedley PL, Jørgensen P, Schlamowitz S, et al. The genetic basis of long QT and short QT syndromes: a mutation update. Hum Mutat 2009; 30: 1486-1511.

8 S7B Nonclinical evaluation of the potential for delayed ventricular repolarization (QT interval prolongation) by human pharmaceuticals. Report number UCM074963. US FDA 2005. www.fda.gov/downloads/drugs/ guidancecomplianceregulatoryinformation/guidances/ucm074963.pdf Date last accessed: July 12, 2016.

9 Vicente J, Johannesen L, Mason JW, et al. Comprehensive T wave morphology assessment in a randomized clinical study of dofetilide, quinidine, ranolazine, and verapamil. J Am Heart Assoc 2015; 4: pii: e001615.

10 Diacon AH, Dawson R, von Groote-Bidlingmaier F, et al. Bactericidal activity of pyrazinamide and clofazimine alone and in combinations with pretomanid and bedaquiline. Am J Respir Crit Care Med 2015; 191: 943-953.

11 Choudhri SH, Harris L, Butany JW, et al. Clofazimine induced cardiotoxicity - a case report. Lepr Rev 1995; 66: 63-68.

Eur Respir J 2016; 48: 1526-1527 | DOI: 10.1183/13993003.01207-2016 | Copyright @eES 2016

From the authors:

We read with great interest the comments by R.S. Wallis on our article describing the first case of extensively drug-resistant tuberculosis (XDR-TB) treated with both delamanid and bedaquiline [1], as recommended by the European Respiratory Society hosted TB Consilium experts [2, 3].

The patient developed QTc (corrected QT or the measure of time between the start of Q wave and the end of $\mathrm{T}$ wave in the heart's electrical cycle (ECG)) prolongation a few days after beginning treatment, that included not only the two new anti-TB drugs but also clofazimine (which, according to Wallis, may have played a role in QTc prolongation). Clofazimine-related cardiotoxicity has been described in a single case-report on an Indian male with leprosy with electrolyte abnormalities treated with clofazimine for 11 months [4]. We agree that there are ongoing safety concerns about QTc prolongation when clofazimine is combined with bedaquiline; safety data remains sparse, although serious life-threatening arrhythmias resulting from this combination have not been described yet.

Our patient had XDR-TB with a single certified active drug, clofazimine, which was the rationale for its inclusion in the salvage regimen. Given the fairly sudden rise in QTc within a week of starting the combination, the TB Consilium experts considered bedaquiline as the culprit for the adverse event. Clofazimine, in fact, requires several weeks to reach the steady state due to its peculiar pharmacokinetics, while bedaquiline at that time was being loaded and, considering its long half-life, the experts recommended to stop it. The patient presented with hypokalaemia during that period, which may have 